\title{
Contribución de las técnicas de medicina nuclear al diagnóstico y localización del hiperparatiroidismo
}

\author{
J. M. JIMÉNEZ-HOYUELA, A. C. REBOLLO, G. I. MESTRE, C. FERNÁNDEZ, \\ E. MONTAÑEZ', J. L. PINZÓN² \\ Servicios de Medicina Nuclear, ${ }^{\prime}$ Traumatología $y^{2}$ Endocrinología. Hospital Universitario \\ Virgen de la Victoria. Málaga
}

DIAGNOSTIC AND LOCALIZATION OF THE HYPERPARATHYROI DISM BY NUCLEAR MEDICINE PROCEDURES

\begin{abstract}
RESUMEN
El hiperparatiroidismo primario viene definido por la hipersecreción de PTH por parte de las glándulas paratiroides. En la mayoría de los casos $(85 \%)$, el origen radica en la existencia de un adenoma de paratiroides, existiendo en algunas circunstancias dificultades para la correcta localización del mismo. Desde hace unos años disponemos de una técnica de medicina nuclear, la gammagrafía de paratiroides con Tecnecio 99m-sestamibi (Tc99m-MIBI), de fácil realización, bajo coste, y excelentes resultados, capaz de proporcionarnos esta información. Presentamos el caso de un paciente diagnosticado de hiperparatiroidismo primario, donde la localización previa a la cirugía del tejido hiperfuncionante, se realizó mediante esta técnica isotópica. Otra exploración de medicina nuclear, la gammagrafía ósea, también contribuyó al correcto diagnóstico de este mismo paciente.
\end{abstract}

PALABRAS CLAVE: Hiperparatiroidismo primario. Gammagrafía de paratiroides. Tecnecio 99m-sestamibi.

\begin{abstract}
Primary hyperparathryoidism is a PTH hypersecretion caused by the parathyroid glands. In most cases (85\%), the origin is to be due to the existence of a parathyroid adenoma, despite the intrinsic difficulty in being localized under certain circumstances. From some time now, we can count with the invaluable help of a nuclear medicine technique, namely the parathyroid scintigraphy with Technetium 99m-sestamibi (Tc99m-MIBI), a technique wich is easy to perform, cheap and with excellent results, and wich additionally can provide us with the above mentioned necesary information regarding location. We present here the case of a patient suffering from primary hyperparatyiroidism, in whom both the disease and the precise location of the hyperfunctioning tissue were identified by means of the parathyroid scintigraphy. Another nucle ar medicine procedure, the one known as bone scintigraphy, also contri buted meaningfully to the correct diagnosis in the same patient.
\end{abstract}

KEY WORDS: Primary hyperparathyroidism. Parathyroid scinti graphy. Technetium 99m-sestamibi

Jiménez-Hoyuela García JM, Rebollo Aguirre AC, Mestre Reoyo GI, Fernández Aguirre C, Montañez Heredia E, Pinzón Martín JL. Contribu ción de las técnicas de medicina nuclear al diagnóstico y localización del hiperparatiroidismo. An Med Interna (Madrid) 2003; 20: 137-140.

\section{INTRODUCCIÓN}

El hiperparatiroidismo primario (HPP) se caracteriza por presentar hipersecrección de PTH. Este hecho es debido en el $85 \%$ de los casos, a la existencia de un adenoma paratiroideo. El curso clínico es variable, pudiendo presentar sintomatología variada: ósea, gastrointestinal, nefrourológica, o permanecer sin síntomas, siendo frecuentemente la hipercalcemia el único hallazgo y el que suele facilitar el diagnóstico. A la hora de localizar el tejido paratiroideo hiperfuncionante, la gammagrafía de paratiroides juega en la actualidad un importante papel, pues permite su localización de una manera fácil, con bajo coste, y con excelentes resultados. Presentamos el caso de un adenoma paratiroideo, en el que técnicas de medicina nuclear facilitaron el diagnóstico (gammagrafía ósea) y localización del mismo (gammagrafía de paratiroides).

\section{CASO APORTADO}

Referimos el caso de un paciente que acude al servicio de urgencias del área de traumatología del hospital, con dolor intenso sobre muslo y rodilla derecha tras haber sufrido de manera fortuita un resbalón con caida al suelo. Se trata de una mujer de 39 años de edad, que como único antecedente de interés refiere dolor en rodilla derecha de 2 meses de evolución, y por el que había iniciado recientemente consulta ambulatoria. A la exploración en urgencias no presentó ninguna clínica de tipo general, ni vasculonervioso. Desde el punto de vista óseo, existía importante dolor en rodilla derecha, así

Trabajo aceptado: 27 de febrero de 2002

Correspondencia: José Manuel Jiménez-Hoyuela García. Servicio de Medicina Nuclear. Hospital Universitario Virgen de la Victoria. Campus Universitario de Teatinos, s/n. 29010-Málaga. e-mail: jmjhoyuela@terra.es 
como tumefacción y deformidad del tercio distal del fémur derecho. Se solicitó analítica de rutina urgente (sangre y orina), así como estudio radiográfico del área afecta. Los resultados de la analítica no mostraron hallazgos de significación. Sin embargo, la radiografía evidenció la existencia de una fractura supra-intercondílea sobre lesión lítica metafiso-epifisaria de fémur derecho. Ante estos hallazgos, se solicita resonancia magnética $(\mathrm{RM})$ de rodilla y fémur derechos con carácter urgente, y se decide ingreso para intervención quirúrgica. La $\mathrm{RM}$ pone de manifiesto la existencia de importante tumoración intraósea, de carácter transmetafisario afectando tanto a la diáfisis como a la epífisis, con extensión a la porción anterior donde infiltra la cortical y se asocia a fractura patológica. Estos hallazgos junto con las caracterísicas de edad, localización, y comportamiento de la lesión sugieren la existencia de tumor de células gigantes. Tras estudio preoperatorio habitual se procede a la cirugía, realizándose reducción, síntesis, biopsia y relleno del defecto con injerto autólogo y heterólogo. Terminado el proceso quirúrgico se realiza interconsulta a oncología, con objeto de iniciar estudio de extensión tumoral. Desde aquí se solicita Tomografía computarizada (TC) toraco-abdominal, así como gammagrafía ósea. El TAC no reveló hallazgos significativos, mientras que la gammagrafía ósea (Fig. 1) entre otros datos evidenció: "zona fría" en extremidad distalcóndilo interno del fémur derecho compatible gammagráficamente con diferentes posibilidades como tumoración ósea, afectación metastásica, etc, así como importante refuerzo hipercaptante de morfología lineal, localizados en huesos largos de extremidades inferiores, que sugiere patología metabólica de base, especialmente compatible con hiperparatiroidismo. Ante este hallazgo se decide realizar
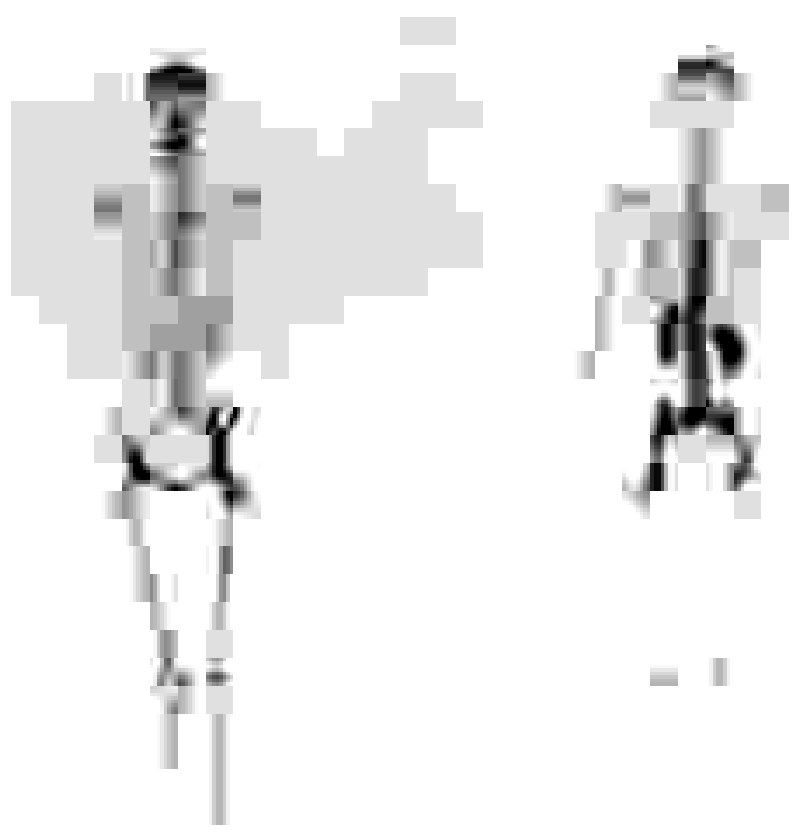

morrwate

lo IIF Hi

Fig. 1. Rastreo gammagráfico óseo realizado con $20 \mathrm{mCi}$ de Tc99mHDP. Destaca evidente "zona fría" en extremidad distal-cóndilo interno de fémur derecho, así como importante hipercaptación en huesos largos, fundamentalmente a nivel cortical, lo que sugiere la existencia de patología metabólica de base. estudio analítico ampliado, incluyendo determinaciones de calcemia y de PTH. Los resultados obtenidos fueron: calcemia de $10,90 \mathrm{mg} / \mathrm{dl}$ $(8,30-9,70)$, y PTH de $1.387 \mathrm{pg} / \mathrm{ml}$ (12-72). Ante estos datos que evidencian la existencia de un cuadro severo de hiperparatiroidismo, se solicita de manera preferente gammagrafía de paratiroides con Tc99m-MIBI para localizar el tejido paratiroideo hiperfuncionante. Lo resultados de la gammagrafía realizada mediante técnica en "doble fase" (Fig. 2), evidenciaron importante foco hipercaptador de morfología redondeada, localizado a nivel del polo inferior del lóbulo tiroideo derecho, indicativo de adenoma paratiroideo derecho. Al día siguiente se realizó intervención quirúrgica, procediéndose a "paratiroidectomía inferior derecha", extirpándose a ese nivel tumoración de 2,5 cm de diámetro con resultado anatomopatológico de adenoma paratiroideo parcialmente quístico, de predominio oncocítico y sin que existan signos de malignidad. En el postoperatorio, desarrolló hipocalcemia ligera (valores de calcemia comprendidos entre 7,3 y $7,8 \mathrm{mg} / \mathrm{dl}$ desde el tercer al octavo día postcirugía), no presentando alteraciones a partir del noveno día. A los tres meses tras

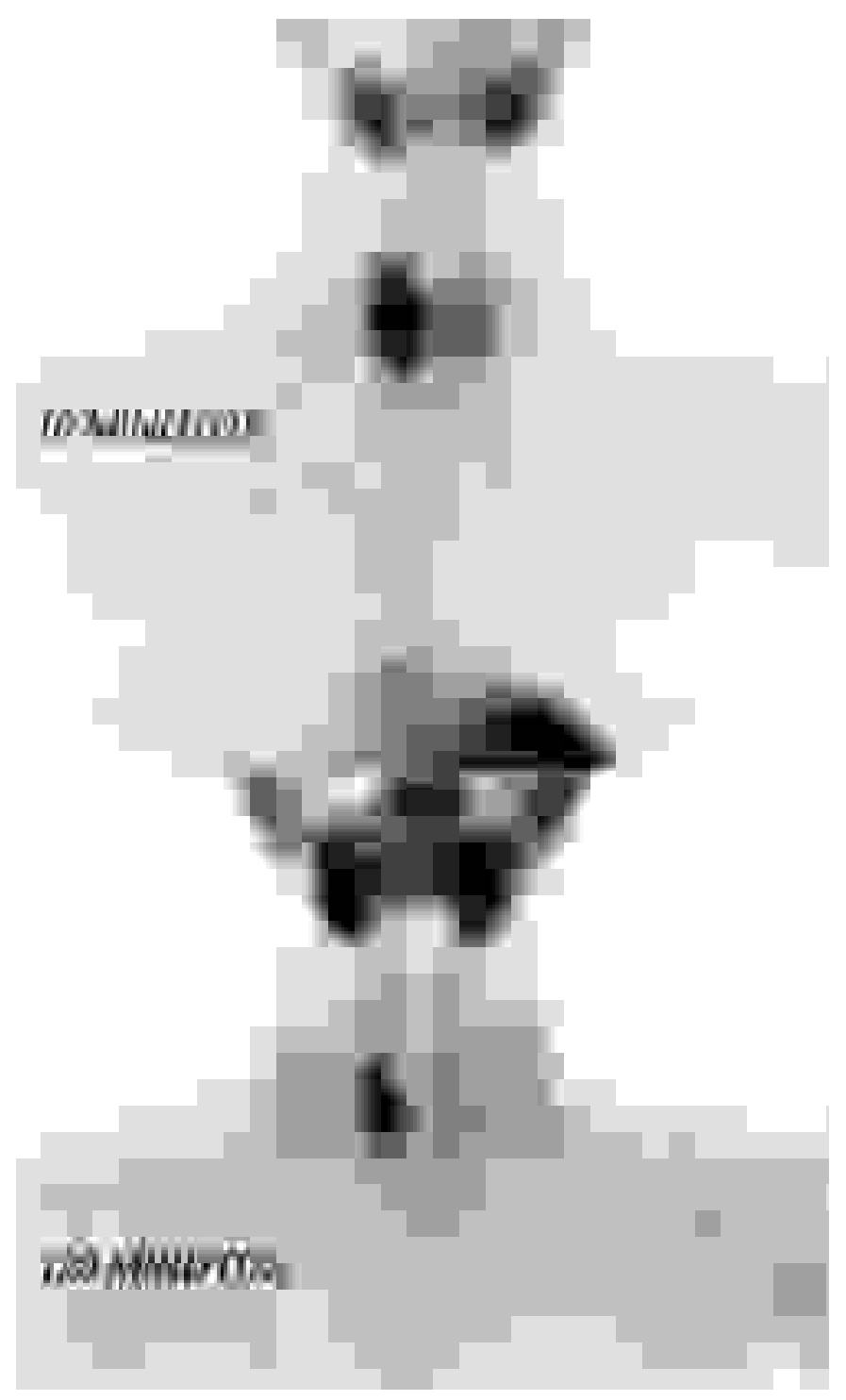

Fig. 2. Gammagrafía de Paratiroides realizada con $20 \mathrm{mCi}$ de Tc99m-M IBI, adquiriéndose imágenes a los 10 minutos y a las 2 horas postinyección del trazador. En la fase precoz existe acúmulo focal del trazador en polo inferior del lóbulo tiroideo derecho, que persiste en la imagen tardía, lo que indica la existencia de adenoma paratiroideo inferior derecho. 
la intervención quirúrgica, los controles rutinarios se encuentran todos dentro de la normalidad (calcemia: $8.52 \mathrm{mg} / \mathrm{dl}$, PTH: 45.3 $\mathrm{pg} / \mathrm{ml}$ ), sin presenar ningún tipo de clínica sugestiva ni otros hallazgos significativos.

\section{DISCUSIÓN}

El hiperparatiroidismo primario (HPP) se caracteriza por la hipersecreción de PTH no controlada por las glándulas paratiroides, persistiendo elevada a pesar de la hipercalcemia presente en estos pacientes. Su etiología es desconocida pensándose en la existencia de un factor etiológico endógeno, que actuaría a nivel de los receptores de las células paratiroideas estimulando de modo anárquico la función. De forma habitual aparece de manera espontánea, pudiendo también tener un origen genético, y formar parte de un síndrome pluriglandular denominado neoplasia endocrina múltiple (MEN). Se conocen dos formas de éste, el tipo I de herencia autosómica dominante y en el que el HPP existe en el $97 \%$ de los casos, y el tipo II asociado a carcinoma medular de tiroides y/o feocromocitoma. La hipersecreción de PTH puede ser producida por un adenoma, por la hiperplasia de las células principales de las paratiroides, o finalmente por un carcinoma. En el 85\% de los casos la causa radica en un adenoma, el cual está más frecuentemente localizado en las paratiroides inferiores. Los casos de hiperplasia se encuentran entre el $10-15 \%$, soliendo afectar a las 4 glándulas. Afortunadamente la presentación de carcinoma es rara, situándose entre el 1-3\%, y suelen diagnosticarse en los casos de hiperparatiroidismo recidivante, existiendo ya en la exploración generalmente invasión local. Desde el punto de vista patogénico, la hipersecreción de PTH provoca como hallazgo fundamental hipercalcemia. El efecto de la hormona al actuar sobre esqueleto y riñón, aumenta la reabsorción de calcio del hueso y del filtrado glomerular, y al actuar estimulando la producción de 1,25 $(\mathrm{OH}) 2 \mathrm{D} 3$ provoca el aumento de la absorción intestinal de calcio. Estas circunstancias afectarán llamativamente al hueso, mostrándose el hueso trabecular fundamentalmente respetado, mientras que el compartimento cortical será el más afectado. El espectro clínico del HPP es amplio y cambiante, existiendo pacientes asintomáticos, y casos de clínica florida. La sintomatología dependerá de múltiples circunstancias, entre las que destacan: la edad del paciente, cuantía de la hipercalcemia, velocidad de instauración de la enfermedad, y precocidad del diagnóstico. Son clásicas la afectación neurológica, digestiva, nefrourológica y ósea. Las lesiones óseas son muy variadas pudiendo manifestarse en la evolución de muy diversas maneras, tales como dolores óseos, deformaciones articulares dolorosas que pueden confundirse con artrosis, ataques de pseudogota por movilización de cristales de pirofosfato cálcico en cartílagos articulares, fracturas óseas que consolidan mal, y la osteítis fibrosa quística $(1,2)$. El diagnóstico de confirmación del HPP se establece poniendo de manifiesto la hipercalcemia, junto con el dato fundamental de la elevación de los valores séricos de PTH. Parte vital dentro del diagnóstico, es la localización del tejido paratiroideo hiperfuncionante con vistas al tratamiento quirúrgico. Existe pleno consenso en la necesidad de practicar técnicas de localización del tejido paratiroideo anómalo en pacientes que deban someterse a un segundo acto quirúrgico por recidiva o persistencia del hiperparatiroidismo. Sin embargo, existe controversia sobre la necesidad de utilización de técnicas de localización en pacientes que vayan a ser intervenidos por primera vez $(3,4)$.
En la actualidad los métodos de localización no invasivos son: ecografía, gammagrafía, tomografía computarizada (TC) y resonancia magnética $(\mathrm{RM})$. La ecografía para la detección de adenomas en el HPP, posee una sensibilidad del $75 \%$. Los falsos negativos son más frecuentes en adenomas de pequeño tamaño, lesiones ectópicas, y cuando coexiste patología tiroidea; son causa de falsos positivos los nódulos tiroideos posteriores exofíticos y las adenopatías cervicales. La hiperplasia presenta estructura y ecogenicidad similar al adenoma, con un tamaño inferior, por lo que es difícil su identificación. El carcinoma no tiene un patrón ecográfico característico. La identificación de glándulas paratiroides normales en pacientes sin hiperparatiroidismo, no constituye una indicación para la cirugía (5). La TC es superior a la ecografía para localizar lesiones paratiroideas de situación retroesofágica y retrotraqueal, así como mediastínicas. Permite además poder realizar la combinación punción-biopsia. Su sensibilidad para localizar tejido paratiroideo anómalo en la primera ciugía es del $79 \%$ y del $60 \%$ en casos de reintervención $(3,4)$. Las limitaciones de la TC para la detección de adenomas incluye artefactos relacionados con la deglución y la respiración, al igual que casos de falsos positivos por adenopatías y estructuras vasculares $(6,7)$. Para la RM el aspecto de los adenomas es variable. Suelen presentar una señal intermedia similar a la del músculo en secuencias T1 y brillante en T2. Las imágenes en T2 son de ayuda para localizar lesiones yuxtatiroideas, pero en mediastino anterior el adenoma brillante desaparece dentro de la señal que produce la grasa tímica. Para el adenoma la sensibilidad es del $75 \%$ en cirugía inicial y del $69 \%$ en caso de reintervención por persistencia del HPP, en la hiperplasia es del $40 \%$. La RM permite una excelente evaluación del mediastino, siendo fuente de errores únicamente la existencia de adenopatías o de lesiones ectópicas múltiples. Para la valoración del carcinoma de paratiroides precirugía ante la sospecha de recurrencia, la RM es la técnica de elección $(4,6,7)$.

Clásicamente la técnica gammagráfica empleada para la detección de tejido tiroideo hiperfuncionante fue la gammagrafía de sustracción Talio-Tecnecio. Se basa en la captación de Talio por parte del tiroides y del tejido paratiroideo anómalo, con la posterior sustracción mediante ordenador de la imagen de Tecnecio que es captado únicamente por el tiroides. Presenta algunos inconvenientes, como la prolongada inmovilización del paciente durante un tiempo considerable, emisión de un tipo de energía no ideal para su detección por parte del Talio, así como una pobre delimitación anatómica (8). Desde hace una década la medicina nuclear dispone de una técnica denominada gammagrafía de paratiroides con Tc99m-MIBI, que por su facilidad de realización y excelentes resultados, es la que hoy se utiliza de manera habitual. Coakley y cols (9) fueron los primeros que sugirieron el uso clínico del Tc99m-MIBI como radiofármaco en la gammagrafía de paratiroides. El Tc99m-MIBI es un complejo catiónico lipofílico que inicialmente se utilizó como agente de perfusión miocárdica. El mecanismo exacto de captación por las paratiroides no está del todo aclarado, sin embargo parece que una serie de factores intervienen de manera simultánea: grado de flujo sanguíneo local, intercambio transcapilar, características químicas como la carga catiónica, carga de la membrana mitocondrial, etc (10). Aunque se han descritos diferentes protocolos, el llamado "doble fase" es el que se realiza de manera habitual. Consiste en usar el Tc99m-MIBI como único trazador, y obtener una imagen gammagráfica paratiroidea precoz (10 minutos postinyección) y otra tardía (a las 2-3 horas), basándose 
en el diferente tiempo de "lavado" del trazador en tiroides y paratiroides, dónde en los casos de hiperfunción es más lento. Se considera un estudio positivo para tejido paratiroideo anómalo, cuando hay acúmulo focal del trazador en la región tiroidea, áreas circundantes o mediastino, que permanece fijo o aumenta en la imagen tardía. Numerosos trabajos han documentado su utilidad (11-14). La sensibilidad antes de la primera cirugía es del $82 \%$ para la detección de adenomas, y del $60 \%$ para glándulas hiperplásicas. En los pacientes reintervenidos por persistencia del hiperparatiroidismo, la sensibilidad se encuentra entre el 70$85 \%$, siendo la especificidad para la detección de lesiones ectópicas del 90-95\%. La coexistencia de patología tiroidea, y lesiones muy pequeñas y de bajo peso (menos de $500 \mathrm{mg}$ ), pueden dificultar la localización.

El caso presentado es el de un paciente con HPP evolucionado que acude al hospital para estudio traumatológico, pero que en este caso la gammagrafía ósea solicitada con el fin de estudiar la extensión tumoral, es la prueba complementaria que permite orientar hacia su patología metabólica subyacente. Aunque la especificidad de la gammagrafía ósea es baja en la enfermedad ósea metabólica, el reconocimiento de patrones anormales propios de ésta, puede ser útil en la interpretación de imágenes atípicas obtenidas en pacientes estudiados por otra sospecha clínica. El estudio gammagráfico óseo puede poner de manifiesto en el hiperparatiroidismo evolucionado, algunas circunstancias tales como hipercaptación craneal, mayor retención ósea del trazador a las 24 horas, así como incremento de la captación en huesos largos (15).

Cuando en el paciente que se presenta se confirma la existencia de valores muy elevados de PTH $(1.387 \mathrm{pg} / \mathrm{ml})$, se decide documentar la existencia de adenoma previamente a la cirugía mediante gammagrafía de paratiroides con Tc99mMIBI. Aunque como hemos dicho, no hay aún consenso sobre la necesidad o no de realizarlo, su uso está cada vez más generalizado y pensamos que debería realizarse de manera habitual al no tener riesgos y poder aportar información de interés. Entre los posibles beneficios del uso de técnicas de imagen previas a la cirugía en pacientes de HPP estarían: exploración quirúrgica unilateral o mínimamente invasiva si se identifica un adenoma, fácil localización de adenomas ectópicos, disminución del tiempo en quirófano y de estancia hospitalaria posterior, y reducción de complicaciones quirúrgicas. En pacientes reintervenidos, hasta el $80 \%$ presentan un adenoma no identificado inicialmente. Se ha comprobado que cuando se realiza imagen preoperatoria, la tasa de éxitos de la cirugía es del $85 \%$ frente al $65 \%$ cuando no se hace. En pacientes reintervenidos el tejido cicatricial dificulta la correcta interpretación de las técnicas morfológicas (ecografía, TC), lo que unido al mayor riesgo quirúrgico de éstos, aconseja disponer también de una técnica de localización de carácter funcional como la gammagrafía. Pensamos que algunos grupos de pacientes se beneficiarían con claridad de la localización prequirúrgica: pacientes reintervenidos, pacientes con problemas diagnósticos, pacientes con características que dificulten la técnica quirúrgica como cuello corto, lesiones tiroideas asociadas o alteraciones de columna cervical, y pacientes de alto riesgo quirúrgico como cardiópatas, etc.

En resumen, la localización de tejido paratiroideo hiperfuncionante mediante gammagrafía con Tc99m-MIBI, es una técnica de fácil realización y disponibilidad, de bajo coste, y que proporciona importante información. Pensamos que la combinación de gammagrafía con Tc99m-MIBI y ecografía es la mejor aproximación para la localización preoperatoria de paratiroides, no sólo en términos de sensibilidad, especificidad y valores predictivos, sino también en relación a los riesgos y costes para el paciente $(16,17)$.

\section{Bibliografía}

1. Bilezikian JP, Silverberg SJ, Gartenberg F, Kim TS, Jacobs TP, Sireis ES. Clinical presentation of prymary hyperparathyroidism. En Bilezikian JP, Marcus R, Levine MA.eds. The Parathyroids, Basic and Clinical Concepts. New York: Raven Press 1994, p. 457-69.

2. Parisien M, Dempster DW, Shane E, Bilezikian JP. Histomorphometric analysis of bone in prymary hyperparathyroidism. En Bilezikian JP, Marcus R, Levine MA.eds. The Parathyroids, Basic and Clinical Concepts. New York: Raven Press 1994, p. 493-503.

3. Norton JA, Brennan MF, Wells SA Jr. Surgical management of hyperparathyroidism. En Bilezikian JP, Marcus R, Levine MA.eds. The Parathyroids, Basic and Clinical Concepts. New York: Raven Press 1994. p. 531-51.

4. Doppman JL. Preoperative localization of parathyroid tissue in primary hyperparathyroidism. En Bilezikian JP, Marcus R, Levine MA.eds. The Parathyroids, Basic and Clinical Concepts. New York: Raven Press 1994, p. 553-65.

5. Grizmann N, Koischwitz D, Rettenbacher T. Sonography of the thyroid and parathyroid glands. Radiol Clin North Am 2000; 38: 1131-45.

6. Hopkins CR, Reading CC. Thiroid and parathyroid imaging. Semin Ultrasound CT MRI 1995; 16: 279-95.

7. Loevner LA. Imaging of the parathyroid glands. Semin Ultrasound CT MRI 1996; 17: 563-75.

8. Fine E. Parathyroid imaging: its current status and future role. Semin Nucl Med 1987; 17: 350-9.

9. Coakley AJ, Kettle AG, Wells CP, O’Doherty MJ, Collins RE. 99mTcsestamibi a new agent for parathyroid imaging. Nucl Med Commun 1989; 10: 791-4.

10. Taillefer R. 99Tc sestamibi parathyroid scintigraphy. En: Freeman LM, ed Nuclear Medicine Annual 1995. New York: Raven Press 1995. p. 51-79.

11. Berná L. 99mTc-Sestamibi en el diagnóstico de localización del hiperparatiroidismo. Rev Esp Med Nuclear 1998; 17: 102-11.

12. Blanco I, Carril JM, Banzo I, Quirce R, Gutierrez C, Uriarte I, Montero A. Double-phase Tc99m sestamibi scintigraphy in the preoperative location of lesions causing hyperparathyroidism. Clin Nucl Med 1998; 23: 291-7.

13. Taillafer R, Boucher Y, Potvin C, Lambert R. Detection and localization of parathyroid adenomas in patients with hyperparathyroidism using a single radionuclide imaging procedure with technetium-99msestamibi (double phase study). J Nucl Med 1992; 33: 1801-7.

14. Caixas A, Berna L, Hernandez A, Tebar FJ, Madariaga P, Vegazo O, et al. Efficacy of preoperative diagnostic imaging localization of technetium $99 \mathrm{~m}$-sestamibi scintigraphy in hyperparathyroidism. Surgery 1997; 121: 535-41.

15. Mari C, Catafau A, Carrió I. Bone scintigraphy and metabolic disorders. QJ Nucl Med 1999; 43: 259-67.

16. De Feo ML, Colagrande S, Biagini C, Tonarelli A, Bisi G, Vaggelli L, et al. Parathyroid glands: combination of $99 \mathrm{mTc}-\mathrm{MIBI}$ scintigraphy and US for demonstration of parathyroid glands and nodules. Radiology 2000; 214: 393-402.

17. Denham DW, Norman J. Cost-effectiveness of preoperative sestamibi scan for primary hyperparathyroidism is dependent solely upon the surgeon's choice of operative procedure. J Am Coll Surg 1998; 186 : 293-304. 